\title{
Soft Artificial Skin with Multi-Modal Sensing Capability Using Embedded Liquid Conductors
}

\author{
Yong-Lae Park ${ }^{1}$, Bor-rong Chen ${ }^{2}$, and Robert J. Wood ${ }^{1,2}$ \\ ${ }^{1}$ Wyss Institute for Biologically Inspired Engineering, Harvard University, Boston, MA 02155, USA \\ ${ }^{2}$ School of Engineering and Applied Science, Harvard University, Cambridge, MA 02138, USA \\ E-mail: ylpark@wyss.harvad.edu, \{brchen, rjwood\}@eecs.harvard.edu
}

\begin{abstract}
We describe the design, fabrication and calibration of a highly compliant mechanism to be used as an artificial skin sensor. The artificial skin sensor consists of multilayered mircochannels filled with a conductive liquid capable of detecting multi-axis strains and contact pressure. A novel manufacturing method composed of layered molding and casting processes was proposed to fabricate a multilayered soft sensor circuit. Silicone rubber layers with channel patterns, cast with 3D printed molds, were bonded to create embedded microchannels, and a conductive liquid was injected into the microchannels. The channel dimensions are $200 \mu \mathrm{m} \times 200 \mu \mathrm{m}$ for strain sensing and $500 \mu \mathrm{m}$ (width) $\times 200 \mu \mathrm{m}$ (height) for pressure sensing. The size of the sensor is $25 \mathrm{~mm} \times 25 \mathrm{~mm}$, and the thickness is approximately $3.5 \mathrm{~mm}$. The prototype was tested with a materials tester and showed linearity in strain sensing and nonlinearity in pressure sensing. The sensor signal was repeatable in both cases.
\end{abstract}

\section{INTRODUCTION}

The development of highly deformable artificial skin with contact force (or pressure) and strain sensing capabilities is one of the critical technologies to advance the area of wearable computing [1] and haptic interfaces in robotics. With tactile sensing, robots are expected to work more autonomously and be more responsive to unexpected contacts by detecting contact forces (or pressures) and locations during activities such as manipulation and assembly. Application areas include haptics [2], humanoid robotics [3], and medical robotics [4]. Different approaches for tactile sensing have been explored. One of the most widely used methods is to detect structural deformation with embedded strain sensors in an artificial skin. Highly sensitive fiber optic strain sensors have been embedded in a plastic robotic finger for force sensing and contact localization [5]. Embedded strain gages have been used in a ridged rubber structure for tactile sensing [6]. Detecting capacitance change with embedded capacitive sensor [7] arrays is another approach for tactile sensing, as shown in a humanfriendly robot for contact force sensing [8]. In spite of their deformability, these example sensing technologies are not truly stretchable and also cannot remain functional at very large strains.

There have been stretchable skin-like sensors proposed with different methods. Strain sensing fabric composites for hand posture and gesture detection has been developed using an electrically conductive elastomer [9]. A stretchable tactile sensor has been proposed also using polymeric composites

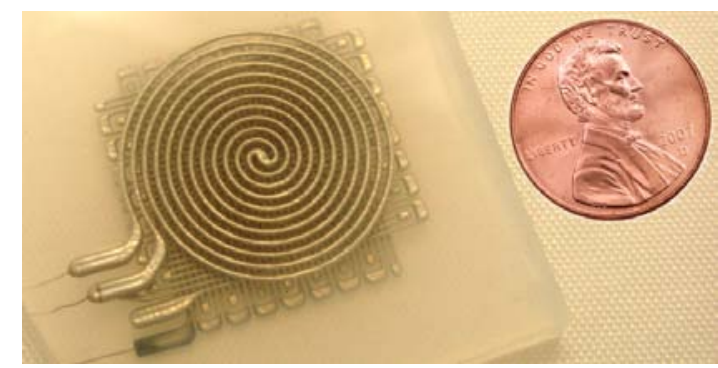

Fig. 1. Soft artificial skin sensor prototype.

[10]. A highly twistable tactile sensing array has been made with stretchable helical electrodes [11]. However, these sensors are not able to remain functional at strains over $100 \%$.

In this paper, we present an artificial robotic skin (Fig. 1) with multi-modal sensing capable of detecting strain and contact pressure simultaneously, designed and fabricated using the concept of hyperelastic strain sensors [12] and pressure sensors [13] with embedded microchannels filled with a liquid metal, eutectic gallium-indium (EGaIn) [14]. The prototype is able to decouple multi-axis strains as well as contact pressure at strains up to $100 \%$.

To our knowledge, this is the first approach to make an artificial skin that provides multi-modal sensing capability with a highly soft and stretchable material and an embedded liquid conductor. The rest of the paper describes the design, fabrication method, and preliminary calibration results.

\section{DESIGN}

The design includes three soft sensor layers made of silicone rubber (see Fig. 2) that is highly stretchable and soft (modulus: $69 \mathrm{kPa}$, shore hardness: 00-30). Layers 1 and 2 have straightline microchannel patterns that are sensitive to axial strains as well as to contact pressure, and Layer 3 has a circular pattern for pressure sensing but is not sensitive to axial strain. Layer 2 is placed on top of Layer 1 with a $90^{\circ}$ rotation for detecting strain along a perpendicular axis. Using the combination of the signals from the three sensors, the device is able to detect and distinguish three different stimuli: $\mathrm{x}$-axis strain, $\mathrm{y}$-axis strain, and z-axis pressure (see Fig. 2(a)). All three sensor layers are connected through interconnects ( $p_{2}$ and $p_{3}$ in Fig. 3 ) between 


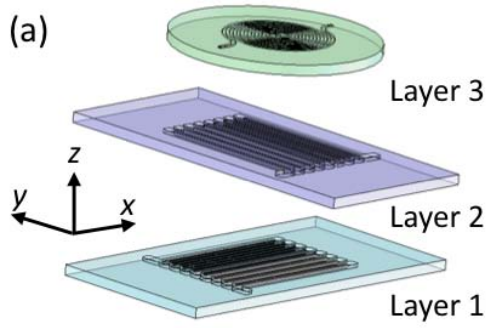

(b)

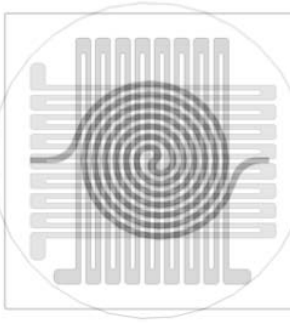

(c)

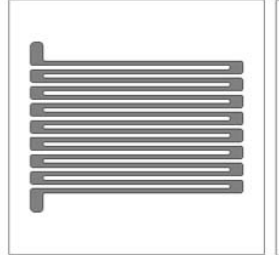

Layer 1

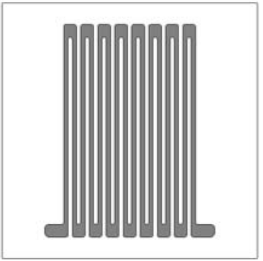

Layer 2

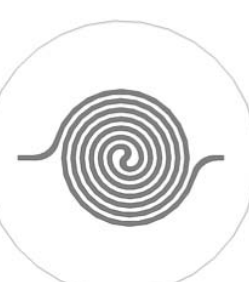

Layer 3

Fig. 2. Design of three sensor layers with embedded microchannels: (a) Exploded view. (b) Assembled view. (c) Each sensor layer design.
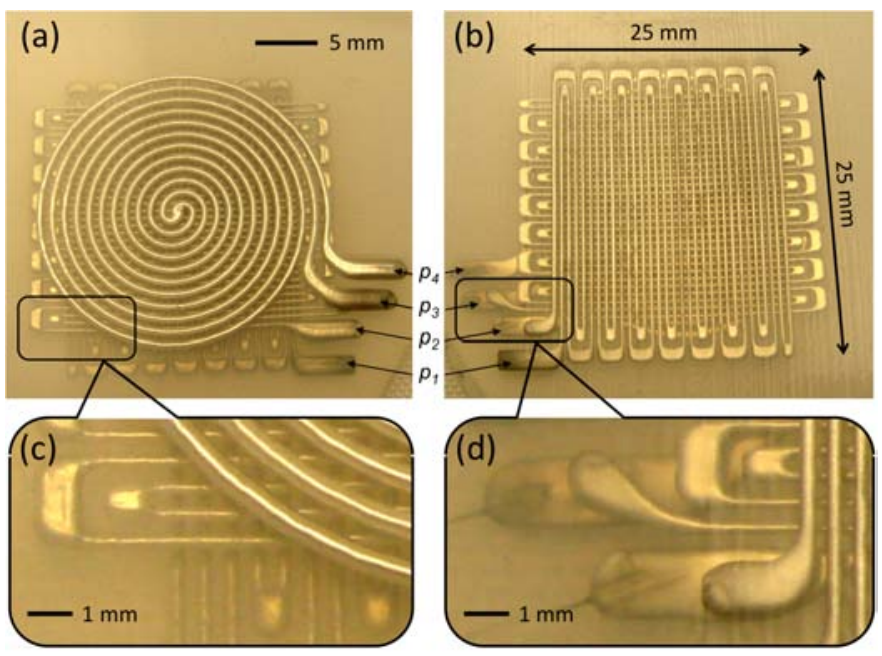

Fig. 3. Actual prototype of the multi-layered soft artificial skin sensor with embedded EGaIn microchannels. (a) Front side of the prototype showing the pressure sensor on top. (b) Back side showing the strain sensor at the bottom. $p_{1}, p_{2}, p_{3}$, and $p_{4}$ are the EGaIn reservoirs for wire connections. (c) Magnified view of the front side showing the multi-layered structure. (d) Magnified view of the interconnects, $p_{2}$ and $p_{3}$, for connecting Layers 1 to 2 and Layers 2 to 3 , respectively.

layers, making one circuit that is electrically equivalent to three variable resistors connected in series.

\section{FABRICATION}

The sensor prototype was fabricated using a layered molding and casting process, as shown in Fig. 4 . The process can be divided into three steps, casting, bonding, and EGaIn injection.

The base material is silicone rubber ${ }^{1}$, which is chosen for its combination of high stretchability (elongation at failure: $900 \%$ ) and ease of casting at room temperature. A relatively

\footnotetext{
${ }^{1}$ EcoFlex0030, Smooth-On, Inc., Easton, PA 18042, USA.
}

(a)

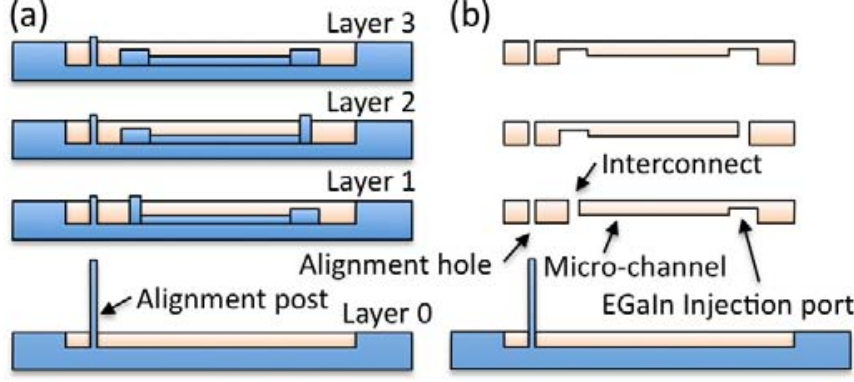

(c)

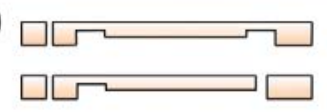

(d)
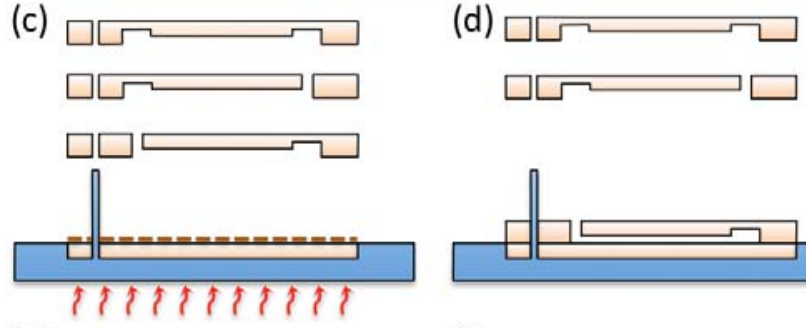

(e)
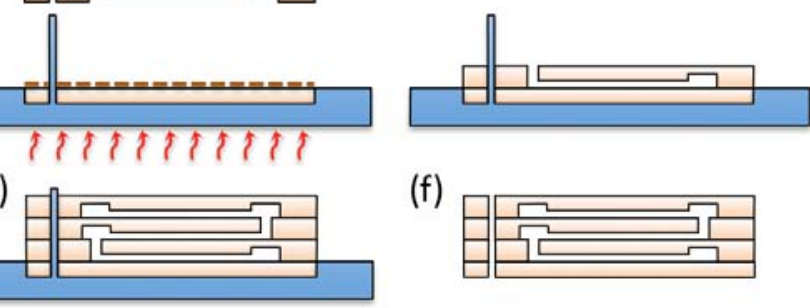

(f)

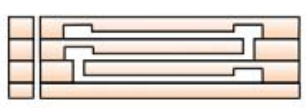

(g)

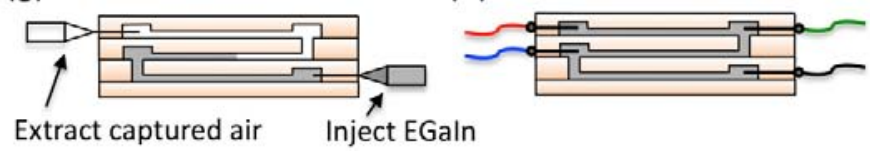

Fig. 4. Fabrication Process. (a) Prepare molds and pour liquid silicone. (b) Remove molds when the silicone cures. (c) Spin-coat Layer 0 (2000 rpm for $50 \mathrm{sec}$.) and partially cure $\left(60^{\circ} \mathrm{C}\right.$ for $1 \mathrm{~min}$.). (d) Bond Layer 1 to Layer 0 . (e) Bond Layers 2 and 3 by repeating spin-coating and partial curing. (f) Remove mold of Layer 0. (g) Inject EGaIn using syringes. (h) Wire connection by inserting electrodes.

low mixed viscosity (3000 cps) is an additional consideration in order to successfully reproduce the features of the mold.

The first step is to cast separate sensor layers (see Fig. 4(a) and (b)). Plastic molds are prepared using a 3D printer ${ }^{2}$, and liquid silicone is poured into the molds. The second step is to bond layers to make a single sensor structure (see Fig. 4(c)-(f)). The cured layers are bonded by spin-coating liquid silicone between layers. Partial curing of the spin-coated silicone prevents the silicone from blocking microchannels. Also, alignment posts in the molds facilitate aligning the interconnects between layers. In the final step, EGaIn is injected into the microchannels and wire connections are made by inserting electrodes (see Fig. 4(g) and (h)). Fig. 5 shows how each layer is bonded to the previous layer with alignment, as described in Fig. 4(c)-(e). In each bonding step, alignment is important to ensure the channel connection between layers through interconnects.

\section{REsults}

A skin prototype was fabricated, as shown in Fig. 3. The channel dimensions are $200 \mu \mathrm{m} \times 200 \mu \mathrm{m}$ for strain sensing

\footnotetext{
${ }^{2}$ Connex500, Objet Geometries Ltd., Billerica, MA 01821, USA.
} 

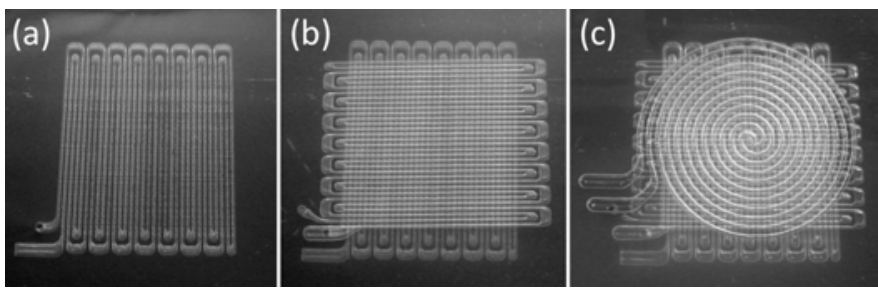

Fig. 5. Photos of microchannel embedded silicone layers showing the layer bonding steps. (a) Layer 1. (b) Layers 1 and 2. (c) Layers 1, 2 and 3.

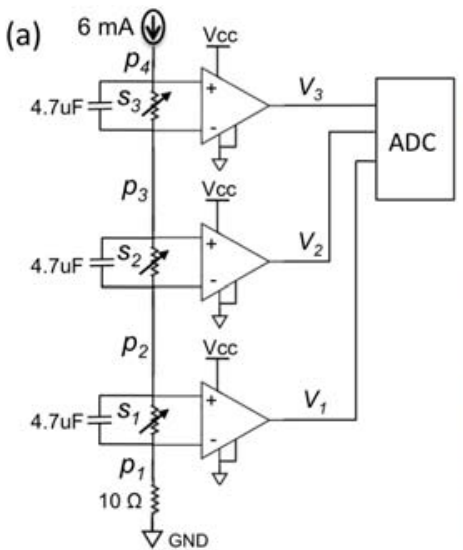

(b)

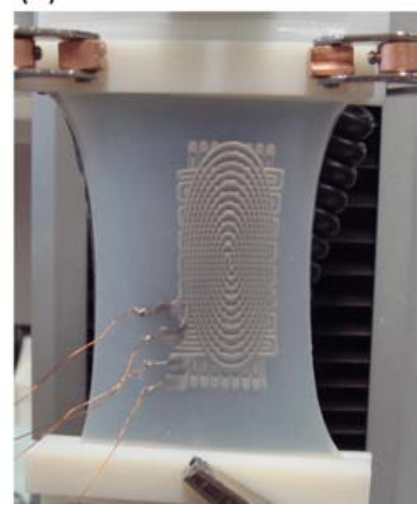

Fig. 6. (a) Circuit diagram to read the sensor signals. $p_{1}, p_{2}, p_{3}$, and $p_{4}$ represent the wire connections in Fig. 3. (b) Example of a calibration experiment: $y$-axis strain.

(Layers 1 and 2) and $500 \mu \mathrm{m}$ (width) $\times 200 \mu \mathrm{m}$ (height) for pressure sensing (Layer 3). The overall size of the artificial skin is $25 \mathrm{~mm} \times 25 \mathrm{~mm}$, and the thickness is approximately $3.5 \mathrm{~mm}$.

Fig. 6(a) shows the circuit diagram to read signals from the three sensor layers. A constant current source generates current that flows through the three sensors in series, creating voltage drops at each sensor layer. The voltage difference across each sensor is amplified by an instrumentation amplifier. The amplified signals are then connected to three analog-todigital conversion ports of a micro-controller to measure the resistance changes.

The sensor prototype was calibrated by applying strains in multiple directions and contact pressure using a materials tester $^{3}$. The prototype was stretched up to $100 \%$ in both $\mathrm{x}$ and $y$ axes for strain sensing (Fig. 6 (b)), and the center of the sensor was compressed up to $60 \mathrm{kPa}$ for pressure sensing. The results showed linearity in strain sensing and nonlinearity in pressure sensing (Fig. 7). However, the sensor signal was repeatable in both cases. Since the signals from the three sensor layers displayed different responses in each experiment, the prototype is able to not only measure the magnitudes of strains and pressure but also distinguish the types of stimuli.

\footnotetext{
${ }^{3}$ Instron 5544A, Instron, Norwood, MA 02062, USA.
}
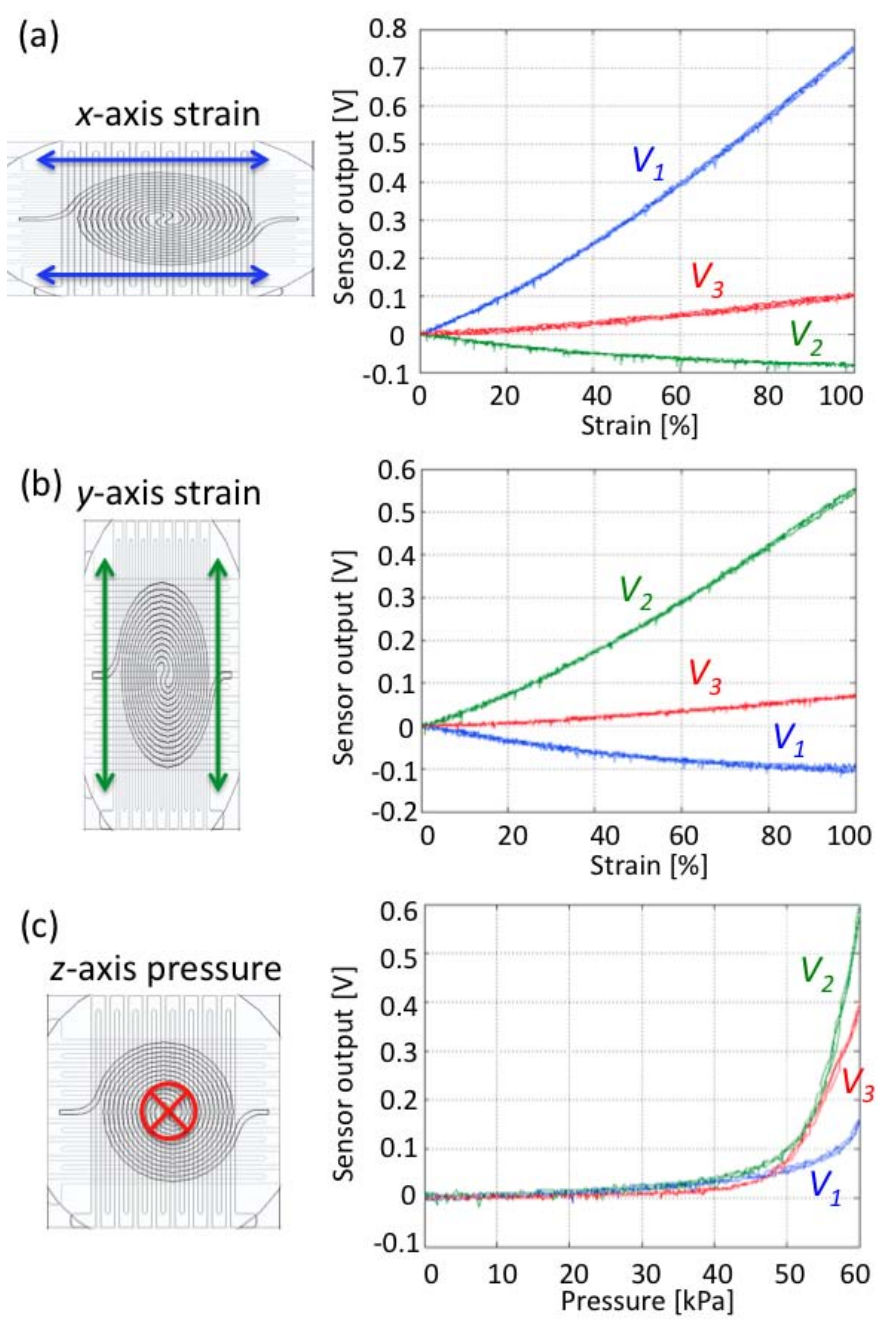

Fig. 7. Calibration results: (a) x-axis strain, (b) y-axis strain, and (c) z-axis pressure.

\section{DISCUSSION}

The main contribution of this work is the design of a multilayered soft artificial skin and the development of a novel fabrication method. The current design provides multi-modal sensing capability requiring no additional sensors. There will be various applications of this technology. Immediate applications may include artificial skins for humanoid robots [3], robotic prosthetics [15], and soft wearable robots [12], [16]. Furthermore, due to the highly flexible and stretchable properties and thin form-factor, this soft skin technology can be directly integrated with any type of soft actuator.

Although the smallest channel size is $200 \mu \mathrm{m}$, limited by the resolution of the $3 \mathrm{D}$ printer used for making molds, this could be further reduced by implementing different manufacturing methods such as micromachined molds [17] and soft lithography [18], [19]. More compact skins could be achieved by reducing the channel size.

While the current design showed linear responses in strain sensing, it gave a nonlinear response in pressure sensing, 
as already modeled and shown in [13]. The nonlinearity in pressure sensing is due to the nonlinear areal reduction rate of the rectangular microchannels when the contact pressure is applied. Improvement on the linearity in pressure sensing is currently being investigated by changing the channel geometry.

During fabrication, EGaIn injection in the multi-layered structure through interconnects makes the fabrication process easy and simple. However, it will not be practical for filling an extremely long channel and/or a higher number of channels. A better manufacturing process without injecting EGaIn is an on-going effort to enable faster and higher volume production.

\section{CONCLUSION}

A highly elastic artificial skin was developed using an embedded liquid conductor. Three hyper-elastic silicone rubber layers with embedded microchannels were stacked and bonded. The three layers contain different channel patterns for different types of sensing such as multi-axial strain and contact pressure. A novel manufacturing method with layered molding and casting techniques was developed to build a multi-layered soft sensor circuit. The calibration results showed linearity in strain sensing and nonlinearity in pressure sensing. However, the sensor signal was repeatable in both strain and pressure responses.

\section{ACKNOWLEDGMENT}

This work was partially supported by the National Science Foundation (award number CNS-0932015) and the Wyss Institute for Biologically Inspired Engineering. Any opinions, findings, and conclusions or recommendations expressed in this material are those of the authors and do not necessarily reflect the views of the National Science Foundation.

\section{REFERENCES}

[1] D. Marculescu, R. Marculescu, N. H. Zamora, P. Stanley-Marbell, P. K. Khosla, S. Park, S. Jayaraman, S. Jung, C. Lauterbach, W. Weber, T. Kirstein, D. Cottet, J. Grzyb, G. Tröster, M. Jones, T. Martin, and Z. Nakad, "Electronic textile: A platform for pervasive computing," Proc. IEEE, vol. 91, no. 12, pp. 1995-2018, 2003.

[2] A. Okamura and M. R. Cutkosky, "Feature detection for haptic exploration with robotic fingers," Int. J. Rob. Res., vol. 20, no. 12, pp. 925938, 2001.

[3] R. Tajima, S. Kagami, M. Inaba, and H. Inoue, "Development of soft and distributed tactile sensors and the application to a humanoid robot," Adv. Rob., vol. 16, no. 4, pp. 381-397, 2002.

[4] P. Puangmali, K. Althoefer, L. D. Seneviratne, D. Murphy, and P. Dasgupta, "State-of-the-art in force and tactile sensing for minimally invasive surgery," IEEE Sens. J., vol. 8, no. 4, p. 371, 2008.

[5] Y.-L. Park, S. C. Ryu, R. J. Black, K. Chau, B. Moslehi, and M. R. Cutkosky, "Exoskeletal force-sensing end-effectors with embedded optical fiber-bragg-grating sensors," IEEE Trans. Rob., vol. 25, no. 6, pp. 1319-1331, December 2009.
[6] D. Yamada, T. Maeno, and Y. Yamada, "Artificial finger skin having ridges and distributed tactile sensors used for grasp force control," $J$. Rob. Mechatron., vol. 14, no. 2, pp. 140-146, 2002.

[7] N. Kirchner, D. Hordern, D. Liu, and G. Dissanayake, "Capacitive sensor for object ranging and material type identification," Sens. Actuators, A, vol. 148 , no. 1, p. $960104,2008$.

[8] J. Ulmen and M. Cutkosky, "A robust, low-cost and low-noise artificial skin for human-friendly robots," in Proc. IEEE Int. Conf. Rob. Autom., Anchorage, AK, May 2010, pp. 4836-4841.

[9] F. Lorussi, E. P. Scilingo, M. Tesconi, A. Tognetti, and D. D. Rossi, "Strain sensing fabric for hand posture and gesture monitoring," IEEE Trans. Inf. Technol. Biomed., vol. 9, no. 3, pp. 372-381, 2005.

[10] L. Ventrelli, L. Beccai, V. Mattoli, A. Menciassi, and P. Dario, "Development of a stretchable skin-like tactile sensor based on polymer composites," in Proc. IEEE Int. Conf. Rob. Biomimetics, Guilin, China, December 2009, pp. 123-128.

[11] M.-Y. Cheng, C.-M. Tsao, Y.-Z. Lai, and Y.-J. Yang, "The development of a highly twistable tactile sensing array with stretchable helical electrodes," Sens. Actuators, A, vol. 166, no. 2, pp. 226-233, 2009.

[12] Y.-L. Park, B. Chen, D. Young, L. Stirling, R. J. Wood, E. Goldfield, and R. Nagpal, "Bio-inspired active soft orthotic device for ankle foot pathologies," in Proc. IEEE/RSJ Int. Conf. Intell. Rob. Syst., San Francisco, CA, September 2011.

[13] Y.-L. Park, C. Majidi, R. Kramer, P. Bérard, and R. J. Wood, "Hyperelastic pressure sensing with a liquid-embedded elastomer," J. Micromech. Microeng., vol. 20, no. 12, 2010.

[14] M. D. Dickey, R. C. Chiechi, R. J. Larsen, E. A. Weiss, D. A. Weitz and G. M. Whitsides, "Eutectic gallium-indium (EGaIn): A liquid metal alloy for the formation of stable structures in microchannels at room temperature," Adv. Funct. Mater, vol. 18, no. 7, pp. 1097-1104, 2008.

[15] H. M. Herr and R. D. Kornbluh, "New horizons for orthotic and prosthetic technology: artificial muscle for ambulation," in Proc. SPIE, vol. 5385, 2004, pp. 1-9.

[16] L. Stirling, C. Yu, J. Miller, R. J. Wood, E. Goldfield, and R. Nagpal, "Applicability of shape memory alloy wire for an active, soft orthotic," J. Mater. Eng. Perform., vol. 20, no. 4-5, pp. 658-662, 2011.

[17] D. S. Kim, H. U. Lee, N. H. Kim, K.-H. Lee, D.-W. Cho, and T. H. Kwon, "Fabrication of microchannel containing nanopillar arrays using micromachined AAO (anodic aluminum oxide) mold," Microelectron. Eng., vol. 84, no. 5-8, pp. 1532-1535, 2007.

[18] D. C. Duffy, J. C. McDonald, O. J. A. Schueller, and G. M. Whitsides, "Rapid prototyping of microfluidic systems in poly (dimethylsiloxane)," Anal. Chem., vol. 70, pp. 4974-4984, 1998.

[19] Y. Xia and G. M. Whitsides, "Soft lithography," Annu. Rev. Mater. Sci., vol. 28, pp. 153-184, 1998. 